\title{
Delivering Beneficial Microorganisms for Corals: Rotifers as Carriers of Probiotic Bacteria
}

\author{
Juliana M. Assis ${ }^{1}$, Fernanda Abreu ${ }^{2 *}$, Helena M. D. Villela ${ }^{1}$, Adam Barno ${ }^{1}$, \\ Rafael F. Valle ${ }^{3}$, Rayssa Vieira ${ }^{3}$, Igor Taveira ${ }^{2}$, Gustavo Duarte ${ }^{1,3}$, David G. Bourne ${ }^{4,5}$, \\ Lone ${\mathrm{H} ø j^{4}}^{4}$ and Raquel S. Peixoto ${ }^{1,3 *}$ \\ 1 Laboratory of Molecular Microbial Ecology, Institute of Microbiology Paulo de Góes, Universidade Federal do Rio \\ de Janeiro, Rio de Janeiro, Brazil, ${ }^{2}$ Laboratory of Cellular Biology and Magnetotaxis, Institute of Microbiology Paulo \\ de Góes, Universidade Federal do Rio de Janeiro, Rio de Janeiro, Brazil, ${ }^{3}$ IMAM-AquaRio - Rio de Janeiro Aquarium \\ Research Center, Rio de Janeiro, Brazil, ${ }^{4}$ Australian Institute of Marine Science, Townsville, WA, Australia, ${ }^{5}$ College \\ of Science and Engineering, James Cook University, Townsville, QLD, Australia
}

OPEN ACCESS

Edited by:

Rodrigo Taketani,

Centro de Tecnologia Mineral, Brazi

Reviewed by:

Gabriel Vinderola,

Facultad de Ingeniería Química, Universidad Nacional del Litoral

(FIQ-UNL), Argentina

Danilo Tosta Souza,

University of São Paulo, Brazi

*Correspondence:

Raquel S. Peixoto raquelpeixoto@micro.ufri.br

Fernanda Abreu

fernandaaabreu@micro.ufri.br

Specialty section:

This article was submitted to

Aquatic Microbiology,

a section of the journa

Frontiers in Microbiology

Received: 21 September 2020 Accepted: 25 November 2020

Published: 15 December 2020

Citation:

Assis JM, Abreu F, Villela HMD, Barno A, Valle RF, Vieira R, Taveira I,

Duarte G, Bourne DG, Høj L and

Peixoto RS (2020) Delivering Beneficial Microorganisms for Corals:

Rotifers as Carriers of Probiotic

Bacteria. Front. Microbiol. 11:608506.

doi: 10.3389/fmicb.2020.608506
The use of Beneficial Microorganisms for Corals (BMCs) to increase the resistance of corals to environmental stress has proven to be effective in laboratory trials. Because direct inoculation of BMCs in larger tanks or in the field can be challenging, a delivery mechanism is needed for efficient transmission of the BMC consortium. Packaged delivery mechanisms have been successfully used to transmit probiotics to other organisms, including humans, lobsters, and fish. Here, we tested a method for utilizing rotifers of the species Brachionus plicatilis for delivery of BMCs to corals of the species Pocillopora damicornis. Epifluorescence microscopy combined with a live/dead cell staining assay was used to evaluate the viability of the BMCs and monitor their in vivo uptake by the rotifers. The rotifers efficiently ingested BMCs, which accumulated in the digestive system and on the body surface after 10 min of interaction. Scanning electron microscopy confirmed the adherence of BMCs to the rotifer surfaces. BMC-enriched rotifers were actively ingested by $P$. damicornis corals, indicating that this is a promising technique for administering coral probiotics in situ. Studies to track the delivery of probiotics through carriers such as B. plicatilis, and the provision or establishment of beneficial traits in corals are the next proof-of-concept research priorities.

Keywords: Beneficial Microorganisms for Corals (BMCs), rotifers, marine probiotics, Brachionus plicatilis, Pocillopora damicornis, delivery, coral reefs, microscopy

\section{INTRODUCTION}

Coral reefs are increasingly impacted by global climate change, which raises the mean sea surface temperature (SST) and the incidence of marine heatwaves (Hughes et al., 2018). Due to increases in the duration and intensity of these thermal stress events, higher rates of coral mortality have been reported globally (Pandolfi et al., 2011; Duarte et al., 2020). Efforts to mitigate the negative effects of global changes on coral reefs have led to the implementation of different strategies in conservation studies. A report published by the United States National Academies of Sciences, Engineering, and Medicine listed several methods of intervention that are currently being 
studied and developed to increase coral resistance and resilience (National Academies of Sciences and Medicine, 2019). One promising approach is the manipulation of different coralassociated microbes to increase host resistance and resilience to stressors (Peixoto et al., 2017; National Academies of Sciences and Medicine, 2019). This strategy relies on key hostmicrobiome symbiotic relationships that can be exploited to increase the fitness of the coral holobiont (Peixoto et al., 2017; Pita et al., 2018; Wilkins et al., 2019). Coral-associated bacteria have been shown to fix nitrogen, degrade polysaccharides, and produce antimicrobial compounds that can inhibit pathogen growth (reviewed by Peixoto et al., 2017). Although the ecological relationships, taxonomic composition, and metabolic pathways of microbial communities associated with corals have been determined (Rohwer et al., 2001; Bourne and Munn, 2005; Sánchez-Quinto and Falcón, 2019), the selection and use of specific microorganisms as probiotics for corals on an ecologically relevant scale is a relatively new field of research (Teplitski and Ritchie, 2009; Santos et al., 2015; Peixoto et al., 2017; Damjanovic et al., 2019; Rosado et al., 2019).

Selection and manipulation of specific members of the resident coral microbiome to mitigate the effects ofthermal stress on the animal health was proposed by Peixoto et al. (2017). The coral-associated bacteria are chosen as beneficial microorganisms involved in the protection, health maintenance, and growth of corals (Rosado et al., 2019). Administering Beneficial Microorganisms for Corals (BMCs) has helped to increase coral resistance against different threats, such as oil contamination, disease, and thermal stress (Santos et al., 2015; Rosado et al., 2019). In these proof-of-concept experiments, a consortium containing BMCs was concentrated and applied directly to corals and the surrounding water, without a biological carrier system (Rosado et al., 2019). Although possible delivery systems have been proposed for closed- and open-water systems (see Peixoto et al., 2017), there is still a lack of studies on potential effective strategies for BMC delivery to corals in aquarium or field settings.

In humans, probiotic foods and beverages are common ways to deliver Lactobacillus species (Roobab et al., 2020) and other beneficial bacteria to improve health (Dunne et al., 1999; Casas and Dobrogosz, 2000; Stolzenbach et al., 2020). Likewise, small organisms such as brine shrimp (Artemia), rotifers, and copepods can be used as vectors for transmitting probiotic bacteria to larger animals in aquaculture, such as fish (Planas et al., 2005, 2006; Sun et al., 2013; Hai, 2015), prawns (Hai et al., 2010), and lobsters (Daniels et al., 2013). Here, we evaluated the potential of the rotifer Brachionus plicatilis (Figure 1 and Supplementary Figure 1) as a vector for delivering BMCs into corals by feeding the rotifers with a previously validated BMC consortium assembled by Rosado et al. (2019), and tracking the uptake of rotifers by the coral Pocillopora damicornis.

\section{MATERIALS AND METHODS}

The steps described in the next topics are summarized in the flowchart shown in Figure 1.

\section{Rotifer and Coral Cultures}

Brachionus plicatilis rotifers and $P$. damicornis nubbins were cultured at the Rio de Janeiro Marine Aquarium Research Center (AquaRio). Rotifers were cultured in natural seawater, at a temperature of $25^{\circ} \mathrm{C}$, salinity of $3.2 \%$, in the dark. Water circulation and oxygenation were produced by bubbling air with an air pump. Using standard culture conditions, rotifers were fed daily with $100 \mathrm{~mL}$ of a culture of the alga Tetraselmis gracilis containing $10^{6}$ cells $\mathrm{mL}^{-1}$. Conversely, before starting the experiment, rotifers were transferred to $1 \mathrm{~L}$ containers with oxygenation produced by bubbling air with an air pump. The container was kept in the dark to avoid the growth of photosynthetic organisms. Rotifers were kept under starvation by culturing them in $0.22-\mu \mathrm{m}$ daily filtered seawater, for a 7 day period.

Corals were kept in tanks containing $100 \mathrm{~L}$ of seawater at $25^{\circ} \mathrm{C}$, salinity of $3.2 \%$, light intensity of $200 \mu \mathrm{mol} \mathrm{m} \mathrm{m}^{-2} \mathrm{~s}^{-1}$, and water recirculation by circulation pumps. Twenty percent of the water in the coral tank system was exchanged every 7 days. Corals were fed daily with $100 \mathrm{~mL}$ of a culture containing 200 rotifers $\mathrm{mL}^{-1}$ under standard culture conditions (i.e., not starved).

\section{BMC Uptake by Rotifers}

The seven strains of bacteria used in the experiment were isolated by Rosado et al. (2019) and stored in the microbial collection of the Microbial Molecular Ecology Lab (MMEL), UFRJ, Rio de Janeiro, Brazil. This consortium was composed of five strains of Pseudoalteromonas spp., isolated from P. damicornis; plus one strain of Cobetia marina and one strain of Halomonas taeanensis, isolated from the artificial seawater surrounding $P$. damicornis. The accession numbers of each isolate and their classifications according to their $16 \mathrm{~S}$ rRNA gene sequences are provided in Supplementary Table 1 .

\section{Localization of the BMC Consortium Associated With Rotifers}

To prepare the BMC consortium assembled by Rosado et al. (2019), individual BMC strains were first grown in Marine Agar (MA) medium to evaluate purity. Sterile inoculation loops were used to pick single bacterial colonies of each strain from the MA plates, and the cells were inoculated into $5 \mathrm{~mL}$ of Marine Broth (MB) medium. Cultures were grown overnight at $26^{\circ} \mathrm{C}$, and $1 \%$ $(\mathrm{v} / \mathrm{v})$ of each strain was individually inoculated into $100 \mathrm{~mL}$ of $\mathrm{MB}$ medium in a $250-\mathrm{mL}$ culture flask and incubated at $26^{\circ} \mathrm{C}$ with agitation of $100 \mathrm{rpm}$ until each culture reached $10^{7}$ cells $\mathrm{mL}^{-1}$. The cells were collected by centrifugation at $12,000 \times g$ for $5 \mathrm{~min}$ at $4^{\circ} \mathrm{C}$ and washed twice under the same conditions with sterile saline $(2.5 \% \mathrm{w} / \mathrm{v} \mathrm{NaCl})$ to remove the culture medium. All cells from each culture were homogenized together, resuspended in a final volume of $40 \mathrm{~mL}$ of saline solution $(2.5 \% \mathrm{w} / \mathrm{v} \mathrm{NaCl})$, and stored at $4^{\circ} \mathrm{C}$ until used in the experiment, which occurred on the same day of the preparation of the cells.

Fluorescence and differential interference contrast (DIC) images were obtained with a Zeiss Axio Imager D2 microscope (Zeiss, Germany). Fluorescence filters used were: (i) Filter set 10 (excitation: BP 332 450-490 nm; beam splitter: FT 510 nm; 


\section{Rotifer delivery system for coral probiotics}
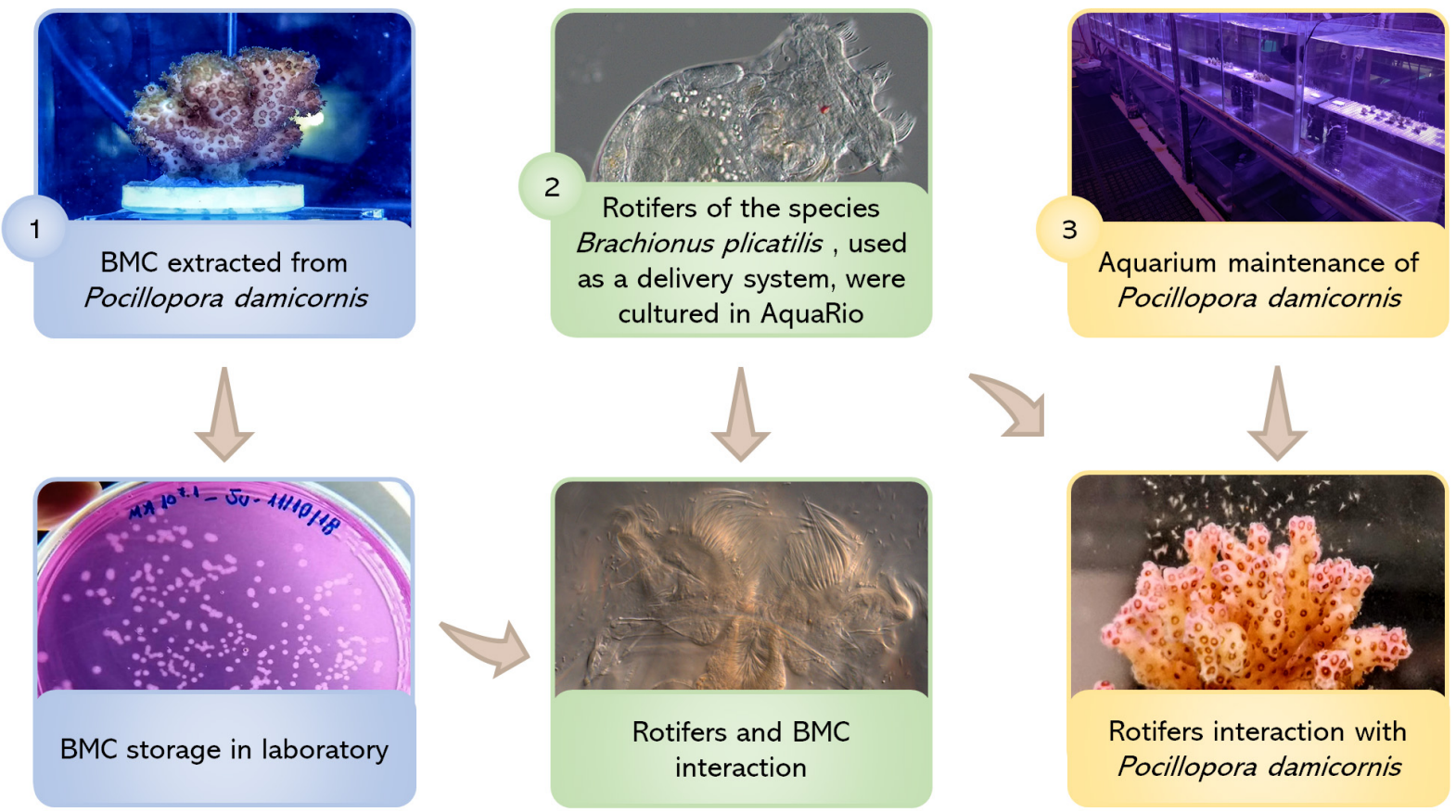

Rotifers interaction with Pocillopora damicornis

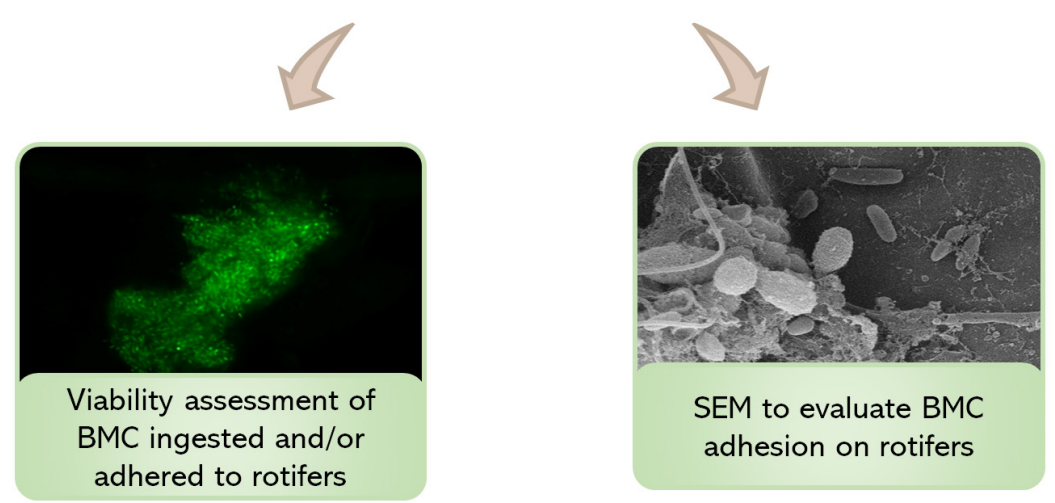

FIGURE 1 | Flowchart showing all treatment steps: (1) BMC strains were isolated from the coral Pocillopora damicornis by Rosado et al. (2019) and deposited in the microbial collection of the Microbial Molecular Ecology Laboratory (MMEL), UFRJ, Rio de Janeiro, Brazil; (2) Rotifers Brachionus plicatilis were cultured at the Rio de Janeiro Marine Aquarium Research Center (AquaRio) and incubated with the BMCs from the MMEL collection. Two different methods were used to assess rotifer-BMC interactions: fluorescence microscopy (using the LIVE/DEAD BacLight ${ }^{T M}$ Bacterial Viability Kit) and scanning electron microscopy (SEM); (3) Nubbins of $P$. damicornis were cultured at AquaRio and transferred to aquarium for the interaction experiment with rotifers $B$. plicatilis. Video and still images of the polyps capturing the rotifers show that the corals were able to take up the rotifers.

emission: LP $515 \mathrm{~nm}$ ); and (ii) Filter set 00 (excitation: BP 546/12; beam splitter: FT 560; emission: BP 575-640 nm).

Based on initial trials, rotifers were starved for 7 days before use in experiments, to limit their autofluorescence by food ingestion. The viability of the rotifers after 7 days of starvation was determined by observing their mobility and cilia beating, using DIC microscopy. The LIVE/DEAD ${ }^{\mathrm{TM}}$ Bacterial Viability Kit (Thermo-Fisher Scientific, United States) was used to stain BMCs, following the manufacturer's protocol. BMCs were washed in sterile seawater to remove excess stain prior to delivery to the rotifers. For BMC-rotifer interactions, the stained BMC homogenized consortium $(100 \mu \mathrm{L}$ of a suspension containing approximately $10^{7}$ cells) was inoculated into a 2$\mathrm{mL}$ polypropylene tube containing $900 \mu \mathrm{L}$ of seawater and a concentration of 70 rotifers $\mathrm{mL}^{-1}$. Promptly after inoculation (around $10 \mathrm{~min}$ ), a $20-\mu \mathrm{L}$ aliquot of BMC-rotifer suspension was mounted on a glass slide, alongside a control with unstained bacteria, and both preparations were observed by means of fluorescence microscopy.

The interactions between rotifers and BMCs were also observed using scanning electron microscopy (SEM). Images were produced from two periods: 4 and $16 \mathrm{~h}$ following incubation 
of the BMC consortium and the rotifer cultures. Starved rotifers without BMC inoculation were used as a control treatment, and performed in parallel with the rotifer-BMC interaction treatments. BEEM-modified capsules (pre-shaped polyethylene molds with hinged lids) with a $100-\mu \mathrm{m}$ polyester mesh filter were used to initiate the sample treatment procedure for SEM. The rotifers were fixed in $2.5 \%$ glutaraldehyde and $0.1 \mathrm{M}$ sodium cacodylate buffer for $1 \mathrm{~h}$ and then washed three times with $0.1 \mathrm{M}$ sodium cacodylate buffer solution and sterile seawater. Samples were post-fixed using 1\% osmium tetroxide for $1 \mathrm{~h}$ at room temperature and washed three times with $0.1 \mathrm{M}$ sodium cacodylate buffer solution and sterile seawater. A series of dehydration washes were performed in ethanol (30, 50, 70, 90, and $100 \%$ concentration) for $10 \mathrm{~min}$ each, with the final $100 \%$ ethanol step repeated three times. Samples were $\mathrm{CO}_{2}$ criticalpoint dried, metallized with gold, and observed in an EVO MA10 scanning electron microscope (Zeiss, Germany) with a voltage between 1 and $30 \mathrm{kV}$.

\section{Coral Uptake of BMC-Enriched Rotifers}

To monitor uptake of rotifers by corals, one nubbin of $P$. damicornis was transferred to a $1-\mathrm{L}$ aquarium containing $0.22 \mu \mathrm{m}$ filtered natural seawater at $25^{\circ} \mathrm{C}$, salinity of $3.2 \%$, and a magnetic stirrer to circulate the water. To normalize the concentration of rotifers $/ \mathrm{mL}$, the culture was filtered using $100 \mu \mathrm{M}$, and reinoculated in $50 \mathrm{~mL}$ of $0.22 \mu \mathrm{m}$ filtered natural seawater. The concentration of rotifers was calculated by counting the number of rotifers in $3 \times 1 \mathrm{~mL}$ replicates of water samples from the previously concentrated samples, using a stereomicroscope (Digilab Zoom binocular stereomicroscope, Brazil). Appropriate dilutions were performed to achieve the desired final concentration of 200 rotifers $\mathrm{mL}^{-1}$. Before added to the coral aquarium, $100 \mathrm{~mL}$ of the culture, containing $\sim 20,000$ rotifers, was washed twice in $0.22-\mu \mathrm{m}$ filtered seawater to clean the maximum of autofluorescence particles that could interfere with the results. Also, the $10-\mathrm{cm}$ coral nubbin acclimatized in the aquarium for $20 \mathrm{~min}$ before the rotifers were inoculated. Video and still images to search for in vivo evidence of rotifer uptake by the corals were taken with a USB digital microscope (Digital USB Microscope, Alloet, China).

\section{RESULTS}

Brachionus plicatilis rotifers cultured under standard conditions (natural unfiltered seawater, at $25^{\circ} \mathrm{C}$, salinity of $3.2 \%$ ) and fed the alga Tetraselmis gracilis displayed autofluorescence signals due to uptake of the algae, which contain photosynthetic pigments that are naturally fluorescent (Figures 2A-C). After 7 days of starvation in $0.22-\mu \mathrm{m}$ filtered seawater, the rotifers displayed no signs of autofluorescence as confirmed by epifluorescence microscopy (Figures 2D-F).

The LIVE/DEAD bacterial assay was used to demonstrate that the members of the BMC consortium, containing five strains of Pseudoalteromonas spp., one of C. marina, and one of $H$. taeanensis (Supplementary Table 1), were viable prior to addition to the rotifer cultures (Figure 3). Using the same staining assay, the uptake of fluorescently stained BMCs by
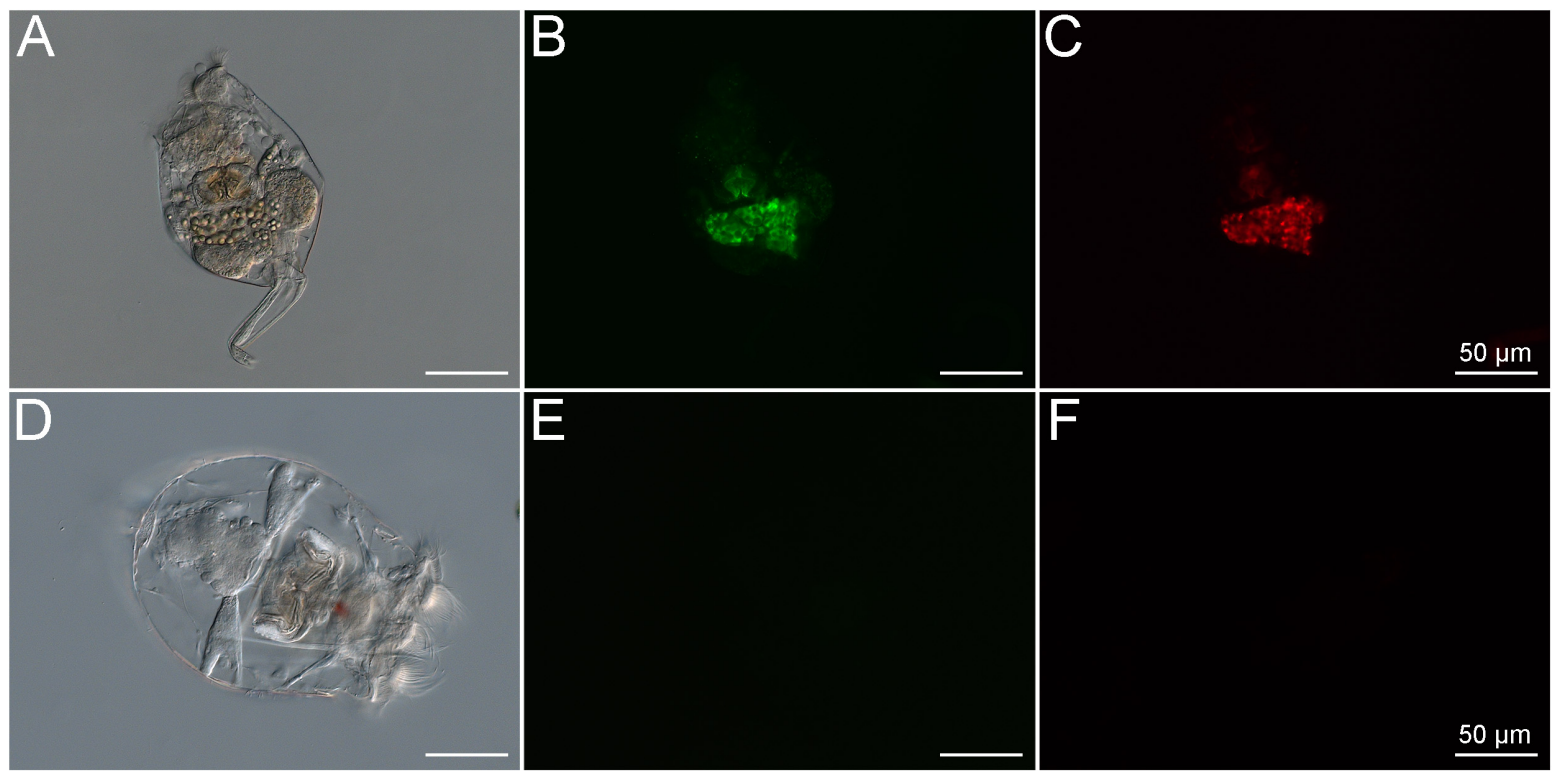

FIGURE 2 | Brachionus plicatilis in standard culture conditions observed in differential interference contrast (DIC) (A) and fluorescence microscopy, using the Filter set 10 (Zeiss, Germany) (B) and the Filter set 00 (Zeiss, Germany) (C). Note that the internal structures of the rotifer show high autofluorescence signals at the same wavelength as both stains used in the LIVE/DEAD ${ }^{\text {TM }}$ BacLight ${ }^{T M}$ Bacterial Viability Kit. This shows that the rotifers take up microbes containing pigments with autofluorescence, probably photosynthetic microorganisms. B. plicatilis cultured after fasting, observed in differential interference contrast (DIC) (D) and fluorescence microscopy using the GFP filter (E) and the Rhodamine filter (F). Note the absence of autofluorescence after starvation. This shows that the starvation period was crucial for the success of the experiment. 

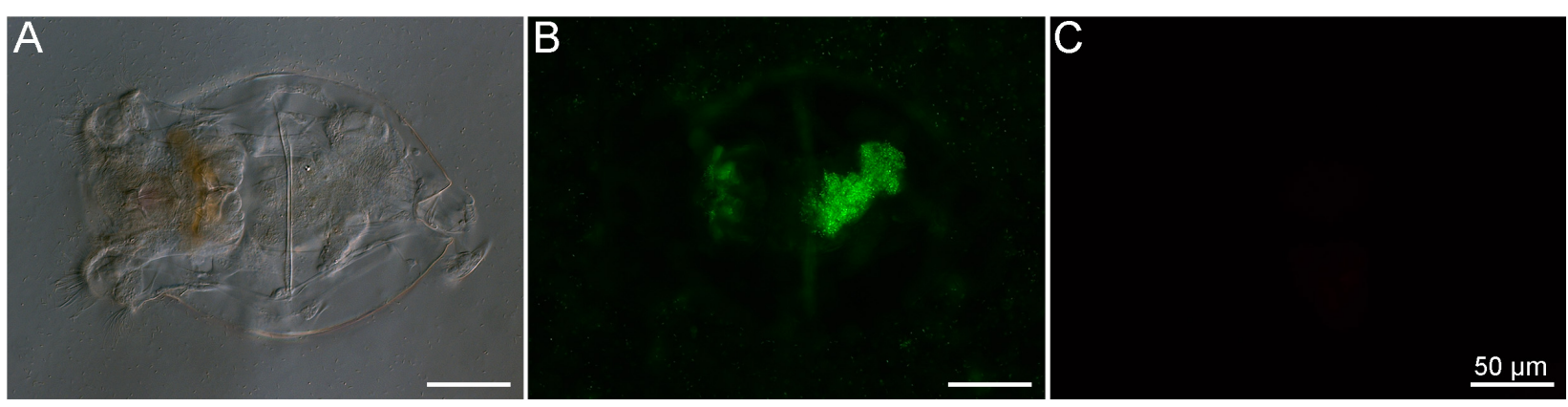

FIGURE 3 | Brachionus plicatilis after incubation with the BMC consortium previously stained with the LIVE/DEAD ${ }^{T M}$ BacLight ${ }^{T M}$ Bacterial Viability Kit observed in differential interference contrast (DIC) (A) and fluorescence microscopy, using the Filter set 10 (Zeiss, Germany) (B) and the Filter set 00 (Zeiss, Germany) (C). Using this kit, viable bacteria cells stain in green while dead cells stain in red. Note that the rotifer ingested a large number of viable bacterial cells (stained in green), resulting in accumulation of stained cells in the stomach and intestine. Bacterial cells also accumulated on the surface of the rotifer, suggesting another possible means of transport of BMC cells.

starved rotifers was observed, using fluorescence microscopy. Large numbers of bacterial cells were ingested within a few minutes after the BMC consortium was inoculated into the rotifer culture (Supplementary Video 1). In parallel, we were able to follow the pathway of BMCs into the rotifer digestive system in vivo, by observing the accumulation of fluorescence in their digestive tract. In addition, we observed strong fluorescence on the external surface of the rotifer body, likely due to the presence of stained BMCs on these surfaces (Figure 3).

Controls (starved, non-BMC-inoculated rotifers) can be visualized with under SEM and show no bacteria interacting with rotifers (Figures 4A,B). Contrarily, SEM revealed the presence of bacteria attached to the corona cilia and rotifer surface after $4 \mathrm{~h}$ of interaction (Figures 4C,D); after $16 \mathrm{~h}$, bacteria were also observed on the body surface (Figure 4E) and corona cilia (Figure 4F).

Finally, rotifers fed with BMCs were added to an aquarium containing nubbins of $P$. damicornis. Using the digital microscope, polyps were seen capturing and ingesting the rotifers containing BMCs (Figure 5 and Supplementary Video 2).

\section{DISCUSSION}

Rotifers are commonly used in aquaculture settings to feed fish and shrimp larvae because they are easy to grow, and, through diet supplementation, they can deliver essential nutrients (Lubzens et al., 1989). Brachionus plicatilis has been widely used for these purposes because of its indiscriminate feeding behavior (Watanabe et al., 1983; Costa et al., 2016) and tolerance to a wide range of salinity levels, making it an ideal organism for transitioning between culture settings and field applications (Lowe et al., 2005).

In this study, we aimed to alter the internal microbiome of $B$. plicatilis to use this species of rotifer as a possible vector for delivery of specific beneficial bacteria to corals. Previous studies have shown that rotifers are hosts to several symbiotic bacteria, but gnotobiotic rotifers are relatively easy to obtain by changing the composition of their diet (Tinh et al., 2006;
Qi et al., 2009). Additionally, it has been shown that Lactobacillus probiotic treatment, delivered by rotifers, positively affected growth, survival, and resistance of western white shrimp in aquaculture (Najmi et al., 2018). Here, we show that the BMC strains isolated from corals seemed to accumulate inside and outside the rotifers body in a stable way, suggesting that rotifers are also a promising vector to deliver probiotics for corals. However, further studies need to be performed to elucidate the viability of the bacteria delivered by the rotifers in the coral body. By optimizing this rotifers-based delivery system and selecting BMC strains with potential to face specific threats, we hope to be able to increase coral resistance against adverse stress conditions, such as ocean warming, diseases, and toxic compounds.

A high proportion of the inoculated BMC consortium likely remained viable throughout the duration of the experiments. The BMC consortium was live/dead-stained prior to inoculation into the rotifer culture, to avoid staining non-BMC bacteria associated with the native rotifers. This confirmed the viability of the BMC bacteria prior to inoculation, although we were unable to determine the viability of BMCs within the rotifers after the uptake. The ingestion rate of the rotifers is indirectly related to digestion and nutrient assimilation rates (Salt, 1987). The 7-day starvation period followed by the high availability of BMC cells potentially increased the rate of bacterial ingestion by the rotifers, and, in parallel, decreased the digestion and assimilation rates (Salt, 1987), thereby prolonging the survival of ingested BMCs.

The bacteria selected to be part of the BMC consortium consisted of five strains of Pseudoalteromonas spp., one of $C$. marina, and one of $H$. taeanensis, that, as a consortium, have been used to mitigate the harmful effects of the coral pathogen Vibrio coralliilyticus in a high-temperature stress scenario (Rosado et al., 2019). Strains of Pseudoalteromonas species have been commonly observed adhering to the surfaces of eukaryotic cells (Holmström and Kjelleberg, 1999; Thomas et al., 2008; Goulden et al., 2013). For example, a probiotic strain of Pseudoalteromonas selectively attached to external surfaces of both the vector organism Artemia and lobster larvae, when introduced as part of a probiotic mixture (Goulden et al., 2013). Here, a strong fluorescent signal consistent with stained bacteria was observed 

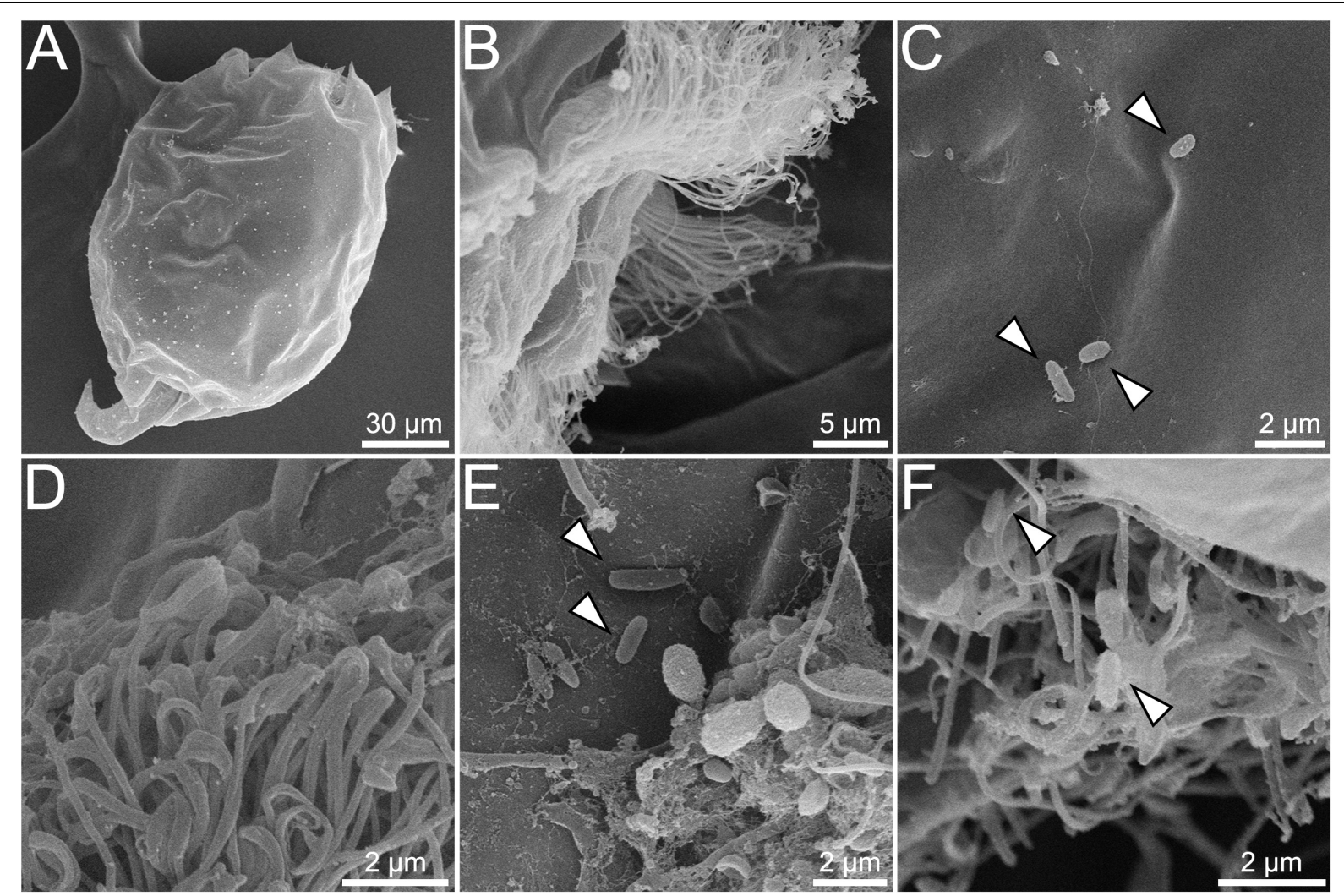

FIGURE 4 | SEM showing interaction between rotifer Brachionus plicatilis and BMCs. Control, with no bacteria visible on the surface of B. plicatilis (A). Higher magnification of control rotifer, showing no bacteria adhered to the corona cilia (B). Rotifer interaction with BMCs after $4 \mathrm{~h}$ (C,D). Higher magnification shows BMCs with rod-shaped bacteria adhered to the rotifer surface (C) (white arrowheads). Corona cilia, showing no BMCs adhered to the rotifer corona cilia (D). Rotifer interaction with BMCs after $16 \mathrm{~h}$, showing rod-shaped bacteria adhered to the rotifer surface (E) and corona cilia (F) (white arrowheads).

on the external surfaces of the rotifers, and these were potentially some of the Pseudoalteromonas spp. strains present in the BMC consortium (Figure 3). Many Pseudoalteromonas strains produce antifouling compounds that can prevent the growth or adhesion of other microorganisms (Holmström and Kjelleberg, 1999; Holmström et al., 2002), including a class of bacteriostatic and amphiphilic anti-Vibrio molecules (Aranda et al., 2012). The Pseudoalteromonas spp. strains included in the BMC consortium may therefore be able to suppress unwanted surface attachment to BMC-enriched rotifers by other opportunistic bacteria before the rotifers are ingested by corals. Pseudoalteromonas spp. strains also protect the gastric region in corals by killing Vibrio pathogens at high temperatures (Tang et al., 2019). All BMC strains used in this study also possess high catalase activity, which can reduce damage from reactive oxygen species (ROS) that are produced in response to stress on the coral (Peixoto et al., 2017; Rosado et al., 2019). Six of the strains (five Pseudoalteromonas spp. and one Halomonas taeanensis) contain enzymes involved in nitrogen fixation or sulfur cycling (Rosado et al., 2019), metabolic processes that may underpin nutrient cycling within the coral holobiont (Raina et al., 2009; Rädecker et al., 2015).

Beneficial Microorganisms for Coral studies are targeted toward delivering microbial communities with traits that promote coral health. Delivery of these BMCs through prey items such as rotifers has proven to be possible, with $P$. damicornis corals readily ingesting the rotifers, representing a promising directed-delivery system for bacteria that provide putative beneficial functions for corals. The rotifer itself also provides a nutritional benefit to stressed corals. For example, Acropora cervicornis increased its feeding rate of the rotifer B. plicatilis under high $\mathrm{CO}_{2}$ conditions, and this raised the coral's total lipid content, which can be a proxy for coral health (Towle et al., 2015). The next steps in establishing this proof of concept consist of determining whether the BMCs delivered through prey items such as rotifers can establish a symbiosis with the coral and provide benefits to the host, and the duration of these associations and benefits.

In summary, this study demonstrated that BMC-enriched rotifers can serve as direct vectors for delivering BMCs to the coral $P$. damicornis. The rotifers freely ingested the BMC consortium, which accumulated in the digestive tract and on the surface. The rotifers were also captured and ingested by the coral polyps, demonstrating the efficacy of using rotifers as a BMC delivery system. These observations represent a step forward in the administration of coral probiotics, which has the potential to increase the persistence and resistance of corals. These findings are also important for probiotic delivery to a wide range of aquaculture species for which rotifers are used as live feed. 


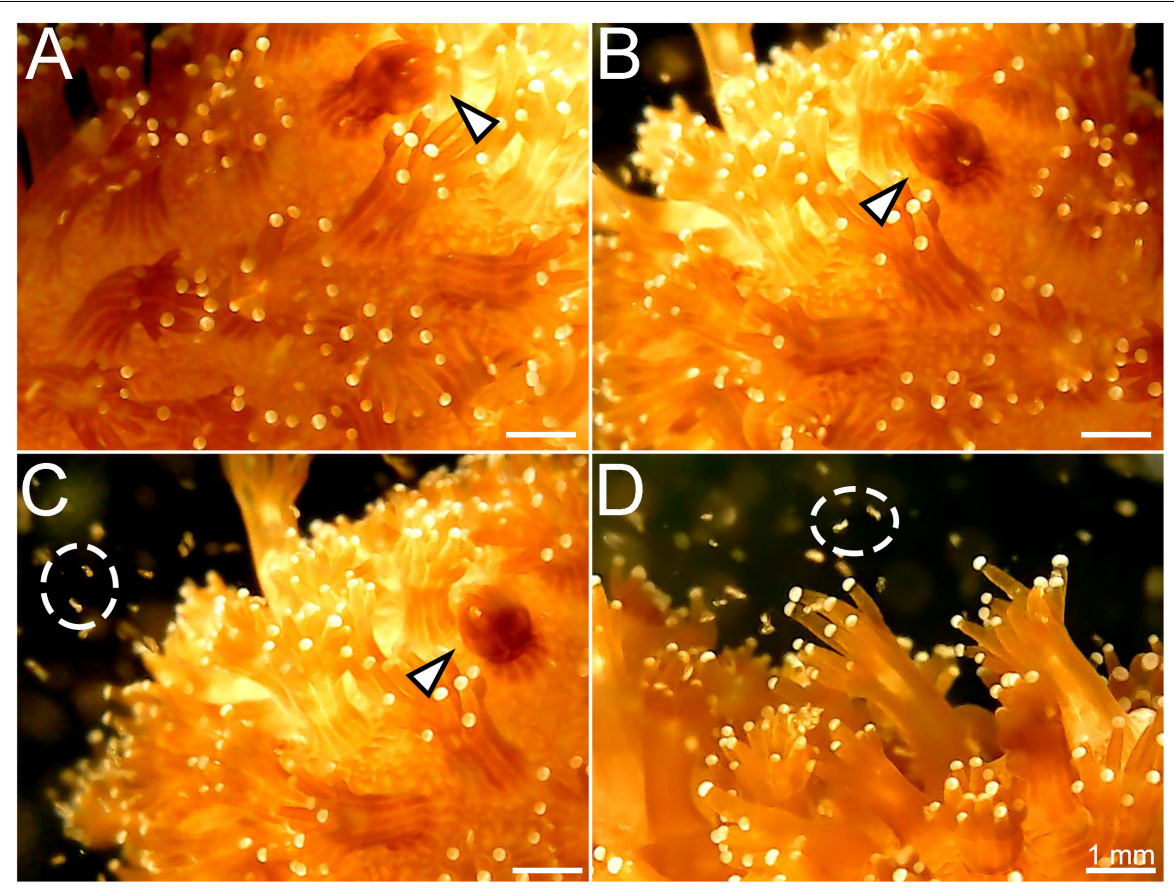

FIGURE $\mathbf{5}$ | Stages of capture of the rotifer Brachionus plicatilis by the coral Pocillopora damicornis. (A) Coral polyp starting to contract after capturing the rotifer (white arrowhead); (B) Polyp totally contracted, engulfing the rotifer; (C) The contracted polyp (white arrowhead) and rotifers swimming around the colony (white circle); (D) Coral polyps widely extended to capture the rotifers (white circle).

Further studies to test this approach in the field and to determine whether BMCs delivered via rotifers are able to establish within the coral microbiome will advance our knowledge of the proper administration of beneficial microorganisms in situ.

\section{DATA AVAILABILITY STATEMENT}

The original contributions presented in the study are included in the article/Supplementary Material, further inquiries can be directed to the corresponding author/s.

\section{AUTHOR CONTRIBUTIONS}

RP, DB, LH, FA, and JMA conceived and designed the study. JMA, RFV, GD, and RV maintained the coral and rotifer cultures. JMA, IT, and FA performed the microscopy experiments. RP, JMA, $\mathrm{FA}, \mathrm{HV}$, and $\mathrm{AB}$ drafted the manuscript. RP provided financial support. All authors were involved in critical revision.

\section{FUNDING}

This manuscript is part of the research project that won the Great Barrier Reef Foundation's Out of the Blue Box Reef Innovation Challenge People's Choice Award, supported by The Tiffany \& Co. Foundation. It also received support from the Graduate Programs of Science (Microbiology) and Plant Biotechnology and Bioprocess Engineering (PBV)/Federal
University of Rio de Janeiro, the National Council for Scientific and Technological Development $(\mathrm{CNPq})$, the Rio de Janeiro Marine Aquarium Research Center, the Carlos Chagas Filho Foundation for Research Support of Rio de Janeiro State (FAPERJ), and the Coordenação de Aperfeiçoamento de Pessoal de Nível Superior - Brazil (CAPES).

\section{ACKNOWLEDGMENTS}

We thank the Great Barrier Reef Foundation, the Tiffany \& Co. Foundation, IMAM-AquaRio, CNPq, FAPERJ, the Graduate Programs of Science (Microbiology) and Plant Biotechnology and Bioprocess Engineering (PBV)/Federal University of Rio de Janeiro for sponsoring this work. IMAM-AquaRio for providing infrastructure, corals, and rotifers. We also thank UniMicro for the support with the fluorescence microscopy experiments. Finally, we thank the National Center for Structural Biology and Bioimaging for the support with the SEM analyses.

\section{SUPPLEMENTARY MATERIAL}

The Supplementary Material for this article can be found online at: https://www.frontiersin.org/articles/10.3389/fmicb.2020. 608506/full\#supplementary-material

Supplementary Figure 1 | Differential interference contrast (DIC) of the rotifer Brachionus plicatilis. The image was taken with a Zeiss Axio Imager D2 microscope (Carl Zeiss, Oberkochen, Germany). Visible morphological features include the corona cilia (purple arrowhead), mouth (blue arrowhead), eye (red 
arrowhead), mastax (yellow arrowhead), intestine (green arrowhead) and anus (orange arrowhead).

Supplementary Table 1 | BMC strains isolated by Rosado et al. (2019) used in this experiment and their respective accession numbers.

Supplementary Video 1 | In vivo fluorescence microscopy showing the fluorescently stained BMCs being ingested by the starved rotifer within 10 minutes

\section{REFERENCES}

Aranda, C. P., Valenzuela, C., Barrientos, J., Paredes, J., Leal, P., Maldonado, M., et al. (2012). Bacteriostatic anti-Vibrio Parahaemolyticus activity of Pseudoalteromonas sp. strains DIT09, DIT44 and DIT46 isolated from Southern Chilean intertidal Perumytilus purpuratus. World J. Microbiol. Biotechnol. 28, 2365-2374. doi: 10.1007/s11274-012-1044-z

Bourne, D. G., and Munn, C. B. (2005). Diversity of bacteria associated with the coral Pocillopora damicornis from the Great Barrier Reef. Environ. Microbiol. 7, 1162-1174. doi: 10.1111/j.1462-2920.2005.00793.x

Casas, I. A., and Dobrogosz, W. J. (2000). Validation of the probiotic concept: Lactobacillus reuteri confers broad-spectrum protection against disease in humans and animals. Microb. Ecol. Health Dis. 12, 247-285. doi: 10.1080/ 08910600050216246-1

Costa, A. P. L., Calado, R., Marques, B., Lillebø, A. I., Serôdio, J., Soares, A. M. V. M., et al. (2016). The effect of mixotrophy in the ex situ culture of the soft coral Sarcophyton cf. glaucum. Aquaculture 452, 151-159. doi: 10.1016/j.aquaculture.2015.10.032

Damjanovic, K., van Oppen, M. J. H., Menéndez, P., and Blackall, L. L. (2019). Experimental inoculation of coral recruits with marine bacteria indicates scope for microbiome manipulation in Acropora tenuis and Platygyra daedalea. Front. Microbiol. 10:1702. doi: 10.3389/fmicb.2019.01702

Daniels, C. L., Merrifield, D. L., Ringø, E., and Davies, S. J. (2013). Probiotic, prebiotic and synbiotic applications for the improvement of larval European lobster (Homarus gammarus) culture. Aquaculture 416, 396-406. doi: 10.1016/ j.aquaculture.2013.08.001

Duarte, G. A. S., Villela, H. D. M., Deocleciano, M., Silva, D., Barno, A., Cardoso, P. M., et al. (2020). Heat waves are a major threat to turbid coral reefs in Brazil. Front. Mar. Sci. 7:179. doi: 10.3389/fmars.2020.00179

Dunne, C., Murphy, L., Flynn, S., O’Mahony, L., O’Halloran, S., Feeney, M., et al. (1999). Probiotics: from myth to reality. Demonstration of functionality in animal models of disease and in human clinical trials. Antonie van Leeuwenhoek. Int. J. Gen. Mol. Microbiol. 76, 279-292. doi: 10.1007/978-94017-2027-4_14

Goulden, E. F., Hall, M. R., Pereg, L. L., Baillie, B. K., and Høj, L. (2013). Probiont niche specialization contributes to additive protection against Vibrio owensii spiny lobster larvae. Environ. Microbiol. Rep. 5, 39-48. doi: 10.1111/1758-2229. 12007

Hai, N. V. (2015). The use of probiotics in aquaculture. J. Appl. Microbiol. 119, 917-935.

Hai, N. V., Buller, N., and Fotedar, R. (2010). Encapsulation capacity of Artemia nauplii with customized probiotics for use in the cultivation of western king prawns (Penaeus latisulcatus Kishinouye, 1896). Aquac. Res. 41, 893-903. doi: 10.1111/j.1365-2109.2009.02370.x

Holmström, C., and Kjelleberg, S. (1999). Marine Pseudoalteromonas species are associated with higher organisms and produce biologically active extracellular agents. FEMS Microbiol. Ecol. 30, 285-293. doi: 10.1016/s0168-6496(99) 00063-x

Holmström, C., Egan, S., Franks, A., McCloy, S., and Kjelleberg, S. (2002). Antifouling activities expressed by marine surface associated Pseudoalteromonas species. FEMS Microbiol. Ecol. 41, 47-58. doi: 10.1016/s0168-6496(02)00239-8

Hughes, T. P., Anderson, K. D., Connolly, S. R., Heron, S. F., Kerry, J. T., Lough, J. M., et al. (2018). Spatial and temporal patterns of mass bleaching of corals in the Anthropocene. Science 359, 80-83. doi: 10.1126/science.aan8048

Lowe, C. D., Kemp, S. J., Bates, A. D., and Montagnes, D. J. S. (2005). Evidence that the rotifer Brachionus plicatilis is not an osmoconformer. Mar. Biol. 146, 923-929. doi: 10.1007/s00227-004-1501-9 of the interaction. The video was recorded with a Zeiss Axio Imager D2 microscope (Carl Zeiss, Oberkochen, Germany) fitted with a GFP filter. (GFP filter details: Zeiss filter set 09 - Excitation: BP 332 450-490 nm; Beam splitter: FT 510 nm; Emission: LP 515 nm).

Supplementary Video 2 | Pocillopora damicornis polyps capturing Brachionus plicatilis rotifers. The video was recorded with a digital USB microscope (Alloet, China).

Lubzens, E., Tandler, A., and Minkoff, G. (1989). Rotifers as food in aquaculture. Hydrobiologia 18, 387-400. doi: 10.1007/bf00048937

Najmi, N., Yahyavi, M., and Haghshena, A. (2018). Effect of enriched rotifer (Brachionus plicstilis) with probiotic lactobacilli on growth, survival and resistance indicators of western white shrimp (Litopenaeus vannamei) larvae. Iran. J. Fish. Sci. 17, 11-20.

National Academies of Sciences and Medicine (2019). A Research Review of Interventions to Increase the Persistence and Resilience of Coral Reefs. Consensus Study Report. Washington, DC: The National Academies Press, doi: 10.17226/ 25279

Pandolfi, J. M., Connolly, S. R., Marshall, D. J., and Cohen, A. L. (2011). Projecting coral reef futures under global warming and ocean acidification. Science 333, 418-422. doi: 10.1126/science.1204794

Peixoto, R., Rosado, P. M., Leite, D. C., de, A., Rosado, A. S., and Bourne, D. G. (2017). Beneficial microorganisms for corals (BMC): proposed mechanisms for coral health and resilience. Front. Microbiol. 8:341. doi: 10.3389/fmicb.2017. 00341

Pita, L., Rix, L., Slaby, B. M., Franke, A., and Hentschel, U. (2018). The sponge holobiont in a changing ocean: from microbes to ecosystems. Microbiome 6:46.

Planas, M., Pérez-Lorenzo, M., Hjelm, M., Gram, L., Fiksdal, I. U., Bergh, $\varnothing$, et al. (2006). Probiotic effect in vivo of Roseobacter strain 27-4 against Vibrio (Listonella) anguillarum infections in turbot (Scophthalmus maximus L.) larvae. Aquaculture 255, 323-333. doi: 10.1016/j.aquaculture.2005.11.039

Planas, M., Pérez-Lorenzo, M., Vázquez, J. A., and Pintado, J. (2005). A model for experimental infections with Vibrio (Listonella) anguillarum in first feeding turbot (Scophthalmus maximus L.) larvae under hatchery conditions. Aquaculture 250, 232-243. doi: 10.1016/j.aquaculture.2005.04.050

Qi, Z., Dierckens, K., Defoirdt, T., Sorgeloos, P., Boon, N., Bao, Z., et al. (2009). Effects of feeding regime and probionts on the diverting microbial communities in rotifer Brachionus culture. Aquac. Int. 17, 303-315. doi: 10.1007/s10499008-9202-x

Rädecker, N., Pogoreutz, C., Voolstra, C. R., Wiedenmann, J., and Wild, C. (2015). Nitrogen cycling in corals: the key to understanding holobiont functioning? Trends Microbiol. 23, 490-497. doi: 10.1016/j.tim.2015.03.008

Raina, J.-B., Tapiolas, D., Willis, B. L., and Bourne, D. G. (2009). Coral-associated bacteria and their role in the biogeochemical cycling of sulfur. Appl. Environ. Microbiol. 75, 3492-3501. doi: 10.1128/aem.02567-08

Rohwer, F., Breitbart, M., Jara, J., Azam, F., and Knowlton, N. (2001). Diversity of bacteria associated with the Caribbean coral Montastraea franksi. Coral Reefs 20, 85-91. doi: 10.1007/s003380100138

Roobab, U., Batool, Z., Manzoor, M. F., Shabbir, M. A., Khan, M. R., and Aadil, R. M. (2020). Sources, formulations, advanced delivery and health benefits of probiotics. Curr. Opin. Food Sci. 32, 17-28. doi: 10.1016/j.cofs.2020. 01.003

Rosado, P., Leite, D. C. A., Duarte, G. A. S., Chaloub, R. M., Jospin, G., Nunes da Rocha, U., et al. (2019). Marine probiotics: increasing coral resistance to bleaching through microbiome manipulation. ISME J. 13, 921-936. doi: 10. 1038/s41396-018-0323-6

Salt, G. W. (1987). The components of feeding behavior in rotifers. Hydrobiologia 147, 271-281. doi: 10.1007/bf00025754

Sánchez-Quinto, A., Falcón, L. I. (2019). Metagenome of Acropora palmata coral rubble: potential metabolic pathways and diversity in the reef ecosystem. PLoS One 14:e0220117. doi: 10.1371/journal.pone.0220117

Santos, H. F. A., Duarte, G. A. S., Rachid, C. T., Chaloub, R. M., Calderon, E. N., Marangoni, L. F., et al. (2015). Impact of oil spills on coral reefs can be reduced by bioremediation using probiotic microbiota. Sci. Rep. 5:18268.

Stolzenbach, S., Myhill, L. J., Andersen, L. O., Krych, L., Mejer, H., Williams, A. R., et al. (2020). Dietary inulin and Trichuris suis infection promote beneficial 
bacteria throughout the porcine gut. Front. Microbiol. 11:312. doi: 10.3389/ fmicb.2020.00312

Sun, Y-Z., Yang, H-L., Huang, K-P., Ye, J-D., Zhang, C-X. (2013). Application of autochthonous Bacillus bioencapsulated in copepod to grouper Epinephelus coioides larvae. Aquaculture 392:44-50. doi: 10.1016/j.aquaculture.2013.01.037

Tang, K., Zhan, W., Zhou, Y., Xu, T., Chen, X., Wang, W., et al. (2019). Antagonism between coral pathogen Vibrio coralliilyticus and other bacteria in the gastric cavity of scleractinian coral Galaxea fascicularis. Sci. China Earth Sci. 63, 157-166. doi: 10.1007/s11430-019-9388-3

Teplitski, M., and Ritchie, K. (2009). How feasible is the biological control of coral diseases? Trends Ecol. Evol. 24, 378-385. doi: 10.1016/j.tree.2009.02.008

Thomas, T., Evans, F. F., Schleheck, D., Mai-Prochnow, A., Burke, C., Penesyan, A., et al. (2008). Analysis of the Pseudoalteromonas tunicata genome reveals properties of a surface-associated life style in the marine environment. PLoS One 3:e3252. doi: 10.1371/journal.pone.0003252

Tinh, N. T. N., Phuoc, N. N., Dierckens, K., Sorgeloos, P., and Bossier, P. (2006). Gnotobiotically grown rotifer Brachionus plicatilis sensu strictu as a tool for evaluation of microbial functions and nutritional value of different food types. Aquaculture 253, 421-432. doi: 10.1016/j.aquaculture.2005.09.006

Towle, E. K., Enochs, I. C., and Langdon, C. (2015). Threatened Caribbean coral is able to mitigate the adverse effects of ocean acidification on calcification by increasing feeding rate. PLoS One 10:e0123394. doi: 10.1371/journal.pone. 0123394

Watanabe, T., Kitajima, C., and Fujita, S. (1983). Nutritional values of live organisms used in Japan for mass propagation of fish: a review. Aquaculture 34, 115-143. doi: 10.1016/0044-8486(83)90296-x

Wilkins, L. G. E., Leray, M., O’Dea, A., Yuen, B., Peixoto, R. S., Pereira, T. J., et al. (2019). Host-associated microbiomes drive structure and function of marine ecosystems. PLoS Biol. 17:e3000533. doi: 10.1371/journal.pbio.300 0533

Conflict of Interest: The authors declare that the research was conducted in the absence of any commercial or financial relationships that could be construed as a potential conflict of interest.

Copyright (c) 2020 Assis, Abreu, Villela, Barno, Valle, Vieira, Taveira, Duarte, Bourne, Høj and Peixoto. This is an open-access article distributed under the terms of the Creative Commons Attribution License (CC BY). The use, distribution or reproduction in other forums is permitted, provided the original author(s) and the copyright owner(s) are credited and that the original publication in this journal is cited, in accordance with accepted academic practice. No use, distribution or reproduction is permitted which does not comply with these terms. 